\title{
Study on the Development of Students' Engineering Qualities in Engineering Physics Teaching
}

\author{
Qing Wang, Jianfeng Dai, Weixue Li \\ Department of Physics, Lanzhou University of Technology \\ Lanzhou 730050, PR China \\ wangqing@lut.cn
}

\begin{abstract}
College physics is an important basic course in university level. In the universities of science and engineering, it is worth further investigating on how to improve the teaching effect in college physics classes, enhance the students' engineering qualities and meet the common requirements for professional trainings and the individual requirements of all students. In this work, some new ideas of innovation were presented and preliminarily attempted based on the modification of training schemes, the updating of teaching contents, the reforming of teaching and assessing methods, in order to develop the students' engineering qualities, establish a scientific ideology and cultivate the capacity for innovation.
\end{abstract}

\section{Keywords-component; formatting; style; styling}

\section{INTRODUCTION}

College physics is an important basic course for education and teaching in colleges and universities, which is compulsory for students of science and engineering. There is no doubt that it plays an important role in cultivating students' abilities and enhancing their comprehensive qualities for engineering teaching. But over the years, its direct applications for engineering practice was relatively neglected [1]. With the expansion of education scale and the diversification of education level in higher education, some new and higher requirements for college physics teaching were put forward according to the change of social environment. In the future teaching, it is worth receiving more attention and further investigating on the reform of college physics teaching, so as to improve the teaching effect in college physics classes, enhance the students' engineering qualities and meet the common requirements for professional trainings and the individual requirements of all students [2-5].

In recent years, with the achieving of national Project of Undergraduate Teaching Quality and Education and Training Plan for Excellent Engineers, the majority college teachers have carried out the reform and innovation on the training targets and plans as well as the teaching contents, methods and strategies, in order to strengthen the cultivating of students' abilities and the engineering ideology for the students of engineering [6]. To cultivate the students' engineering qualities is to enable them to establish the correct engineering thinking and engineering ideology, to think independently in varies engineering applications, to simplify the practical problems so as to solve the main problems in the intricate engineering applications, to develop the capacity of solving practical engineering problems in a feasible, economical and simple way, and to improve themselves in continuous studying and engineering practicing, becoming qualified senior engineers and technicians. The following is the author's thoughts on the development of students' engineering qualities in college physics teaching.

\section{A. For Different Training targets, Making Different Teaching Programs and Implementation Plans}

College physics science and engineering is a general education courses, according to the non-physical class science and engineering disciplines university physics teaching college physics course content system includes Newtonian mechanics, electromagnetism, thermodynamics and kinetic theory, several parts of the optics, the special theory of relativity and quantum physics basis. The entire contents of the system should be highlight the fundamental focus, and stress difficult problems on the basis content. College physics course content, the limited number of hours, to adapt to different training goal, according to the nature and characteristics of the various professional disciplines, the professional engineering school can be divided into three types of modules: machinery, power and materials chemical class. Classroom teachers can do the appropriate adjustments, when teaching different professions and content in class. Power module for machinery, including mechanical engineering and automation, industrial engineering, packaging engineering, civil engineering, building environment and equipment engineering, energy and power engineering, which is teaching focus on mechanical and electrical. For power class module, including electrical engineering of automation, communications of computer science and technology, increase the number of semiconductor knowledge and laser contents, but focus on electromagnetism and modern physics. For materials chemical class module, including materials science and engineering, chemical process, environmental engineering, food engineering and pharmaceutical engineering, it should be focus on atomic physics knowledge. Finally, Teaching to pay attention to the explanation of the physical ideas to reduce cumbersome calculation and derivation of the mathematical, it is enhancing engineering applications and stimulating innovation to play an important role in the 
development of high-tech, with due regard to the depth to keep the cutting-edge and advanced.

\section{B. According to the Different Professional Engineering Background in Engineering and Technical to Analysis of the Physical Problems}

We reached a consensus with the development of the reform of higher education that university physics courses are the purpose in the training objectives. For an engineering-based University of Science and Engineering how to let students study the physical knowledge of the system, reflect the overall picture of the physical world, understand the current levels and trends of the development of physical theory in the limited time, while combining with the professional engineering background at the same time to train students of engineering and technical analysis capabilities of the physical problems, it is an important aspect for the reform of engineering physics teaching content to improve the literacy of students' engineering, according the actual situation to increasing the principles of physics in modern engineering technology. we selected 30 physical principles and engineering problems are due to the limitation of the number of hours, 30 engineers and technicians in the physical is not possible in the classroom to explain one by one, according to the classification of the three modules, 30 issues throughout the system of the physical content for different professional engineering background. For example Mechanical Engineering, the increase in the mechanical part of the application analysis, such as the conservation of angular momentum and inertial navigation principles, standing wave phenomena and stringed instruments tuning principle, the law of conservation of momentum and rocket flight, the rest of the engineering applications according to the teaching talk less about the progress or not. For Electrical Specialized increase electromagnetics application analysis, such as the currentcarrying cylindrical magnetic field distribution and magnetic particle detection technology, the principle of electrostatic induction and tip discharge electrostatic dust, electrostatic shielding and the principle of electromagnetic shielding device, eddy current principle, electromagnetic heating technology, dielectric polarization and microwave heating technology. For materials discipline, increase the thermal and optical applications, such as cycle chillers and heat pumps, artificial birefringence photoelastic effect, optical rotation phenomena and sugar meter, X-ray diffraction and X-ray diffraction analysis. In addition, teachers based on professional characteristics what succinctly speak part of the problem in the teaching process, some of the problems can also be completed by Students in class by mean of a short essay as usually results recorded in the evaluation.

Between research and teaching constantly exchange. Therefore, the specific combination of specific knowledge and teachers' physics research work also an important way to cultivate students' engineering quality in the university. It is heart of teaching to find the historical links between the research work and college physics and explore old and unknown knowledge. To play an unexpected effect for promoting university physics teaching effectiveness for achieving the organic combination of scientific research and the teaching of physics, not only the old classical theory taught in the application, but also give students a comprehensive understanding of the latest research in the field [7].

\section{Introducing Diverse Teaching and Assessment Methods to Cultivate students' Engineering Literacy}

The principles of technology physics play an important role in training of scientific literacy for engineering and innovative thinking. In order to strengthen the quality of the engineering in college physics teaching, we introduce the diverse teaching and assessment methods to improve the literacy of engineering.

First, the introduction of a typical engineering application not only enrich the classroom teaching but also contacted the project instance, so that students fully understand the importance of physical theory and enhanced physical interest and enthusiasm for learning. Second, according to the characteristics of the different professions, teachers specified topics in order to self-study by the students. Third, through the physics demonstration experiment to establish the awareness of students' engineering and train create ability. The school has established a high-level demonstration laboratory in order to cultivate students. Fourth, a profession of physical knowledge and skills competition held annually to develop students' ability to innovate in practice. Fifth, use of the school's innovative education programs platform for the application of the principles in engineering technology innovation. Classical physics in engineering technology has a wide range of teaching after years of research and teaching practice, we have prepared the physical principles and engineering technology to select of ten thematic as an innovative educational curriculum content, to guide students concerned about the physics and production technology applications, to carry out a research study activities, student teachers can be independently completed under the guidance of the topics, developing the ability to guide students and explore the future development of new knowledge and information to help students predict, foresight and vision of future things occurrence so as to nurture students' creative thinking and innovative ability. Sixth, taking into account the purpose of university physics teaching and curriculum for students to make the appropriate adjustments and learn pressure test method. Integrated use of a variety of assessment methods to establish a rational evaluation mechanism, use of normal results $(30 \%)$ and ending closed book exam (70\%) combined ratings. Accounted for $30 \%$ of the normal results include classroom performance $10 \%$, the job completed $10 \%$ and the book report $10 \%$. Class performance by the attendance and questions performance to give appropriate score based on total attendance of students for a semester, which constrain some slack students to lectures and urge students, teachers test students' understanding of the relevant theoretical knowledge and understanding of scientific and technological frontiers of knowledge by teaching in the classroom, it can improve the 
students' attention and stimulate students' enthusiasm for learning and create a interactive classroom teaching environment. Job completion of homework scores and classroom quiz results allow teachers to understand the students. According to the characteristics of the different professions requires students to write a book report around the content, the student must be access relevant information for enhancing their theoretical knowledge, but examines can enhancing the students' self-learning ability and creative thinking ability. Combine theoretical and practical are able to inspire students to learn physics keen interest. The end of a closed book exam used to test them of college physics course. Through a variety of assessment methods can help students to establish physical ideas and check their ability of the knowledge, that not only can achieve the purpose of college physics teaching, but also reduce students' exam pressure and popular with students.

\section{REFERENCES}

[1] Li Fuquan, Zhang Xiaochun, Zhao Zhizhong, around the culture of engineering consciousness and innovation ability. Deepen the reform of college physics teaching, China Higher Education Research, 2001 (2) 66-67.

[2] Wang Jialing, Du Xin, University of Engineering Physics Teaching Reform of Higher Studies, 2009 (7) 173-174.

[3] Lu Xiaolu, recorded Reflections university physics teaching physical quality training, extracurricular education - Subject Education, 2010 (4) 113.

[4] Fang Limei, Liu Genwang, the culture of college physics teaching scientific literacy and scientific inquiry ability, experimental techniques and management, 2008 (10) 134-136.

[5] Li Gailan, on the way to improve the quality of university physics teaching, educational theory and practice, 2008 (9) 61-62.

[6] Liu Huimeng, Huang Ronghua, Wang Zhaowen, strengthen engineering literacy ability to focus on training, teaching and research, 2012 (5) 62-63.

[7] Ning Changchun, Suo Lang Sam, introduced to the research work of integration to promote the teaching of college physics, physics, 2012 (8) 39-43. 